\title{
UNA DECISIÓN CONTROVERTIDA: CONTRATO DE PLAZO FIJO Y FUERO MATERNAL ${ }^{*}$
}

\author{
ROBERTO CERÓN REYES \\ FELIPE PEROTI DIAZ \\ Universidad de Chile
}

\section{INTRODUCCIÓN}

El propósito de este comentario de jurisprudencia es presentar y analizar el razonamiento e implicancias normativas en torno a la tensión existente entre el contrato de plazo fijo y el fuero maternal, y su incidencia en la tutela de los derechos fundamentales. Para ello, iniciaremos el análisis (2) con una breve exposición de los hechos y la decisión del tribunal, para después abocarnos al (3) estudio del razonamiento de la Corte, para terminar con una (4) conclusión que pretende presentar los posibles lineamientos que den con una solución aceptable al caso.

\section{DESCRIPCIÓN DE LOS HECHOS Y DECISIÓN DEL TRIBUNAL}

La recurrente, Elizabeth Redel Troncoso, fue contratada como vendedora de tienda comercial, bajo la modalidad de un contrato de plazo fijo, desde el 22 de noviembre hasta el 31 de diciembre de 2006, firmándose el finiquito el 31 de enero de 2007. Posteriormente, el 31 de marzo de 2007, la trabajadora fue informada que se encontraba embarazada desde el 28 de diciembre de 2006 (Considerando tercero). Una vez conocido el estado de gravidez y el requerimiento efectuado por personal fiscalizador de la Inspección del Trabajo, el empleador se allanó a poner término a la separación indebida y a pagar las remuneraciones y cotizaciones previsionales por el tiempo de la separación. Después de esa visita de fiscalización y hecha la reincorporación fruto de la mencionada fiscalización, la recurrida procedió a desvincularla nuevamente (Considerando cuarto).

Con fecha 27 de octubre de 2008, la Primera Sala de la Ilustrísima Corte de Apelaciones de Temuco, en los autos caratulados Redel Troncoso, Elizabeth / Nulidad de despido, decidió anular el despido de la recurrente, al señalar que no cumple los requisitos del artículo 174 del Código del Trabajo, relativos al fuero laboral, por encontrarse la recurrente embarazada.

\footnotetext{
* Roberto Cerón. Egresado de Derecho. Universidad de Chile. Ayudante de Derecho del Trabajo y de Historia del Derecho en la misma universidad. Felipe Peroti. Egresado de Derecho. Universidad de Chile. Ayudante de Derecho Constitucional y de Historia del Derecho en la misma universidad. Agradecemos las generosas observaciones realizadas por el profesor ayudante de Derecho del Trabajo Sr. Felipe Westermeyer.
} 


\section{RAZONAMIENTO DEL TRIBUNAL}

Al fundamentar su decisión, la Corte estructura su argumentación en base a los siguientes antecedentes de hecho y de derecho. En primer lugar, señala:

“... Que la trabajadora aforada por causa de embarazo... goza de una situación de protección especial dirigida a la protección de la maternidad y del que está por nacer, en que es necesario cautelar su entorno, ante la evidente indefensión de ambos..., alcanzándoles la extensión del derecho a la seguridad social contemplado en el art. $19 \mathrm{~N}^{\circ} 18$ de la Constitución Política, desde el punto de vista de la trabajadora embarazada y desde el punto de vista del ser que está por nacer su situación se encuentra protegida justamente por el art. $19 \mathrm{~N}^{\circ} 1$ inciso $2^{\circ}$ de la Constitución Política". (Considerando primero).

En segundo lugar, analiza la protección que envuelve el fuero del artículo $174 \mathrm{del}$ Código del Trabajo, al indicar:

“... El fuero maternal, en los términos como se encuentra establecido en el Código del Trabajo, consiste en la imposibilidad a que el empleador pueda poner término al contrato, con su voluntad, sin que medie una autorización judicial previa, autorizándolo. Obviamente, entonces, queda fuera del impedimento todos aquellos casos en que el contrato termina por una causal distinta a la manifestación de voluntad del empleador, casos en los cuales la terminación procede, aun en estado de embarazo como sería el caso del contrato de plazo fijo, que concluye por el vencimiento del plazo y no por despido del empleador..." (Considerando primero).

En tercer lugar, ante la interpretación de los artículos 174 inciso primero y el artículo 201, ambos del Código del Trabajo y frente a la temporalidad definida del contrato de plazo fijo expresa:

“... Si fuese procedente entender que el contrato de plazo fijo termina, aun con embarazo iniciado durante su vigencia, ya que la terminación del contrato se debe a la llegada del plazo y no a la voluntad o despido del empleador. ¿̨Cómo se explica que, a renglón seguido, el legislador exija autorización judicial previa del juez competente quien la podrá otorgar solo en dos casos, siendo el primero de ellos el vencimiento del plazo convenido? Ante estas situaciones contradictorias, causadas por la deficiente redacción de los textos legales, el juez debe apreciar la prueba con las reglas de la sana crítica, y resolver el conflicto y ejercer la autoridad conforme a lo dispuesto por el art. 76 de la Constitución Política, es decir "ni aun por falta de ley que resuelva la contienda o asunto sometido a su decisión..." (Considerandos segundo y tercero).

En cuarto y último lugar, atendida la oportunidad de decisión que debe, necesariamente, efectuar el tribunal de segunda instancia afirma “... Para ello esta Corte estima oportuno considerar la jerarquía de los principios y garantías constitucionales interpretan- 
do aquellos que otorgan protección a la seguridad social y de la vida del que está por nacer" (Considerando tercero). Al efectuar el análisis de ponderación entre los derechos constitucionales arriba señalados y el principio de la autonomía de la voluntad que envuelve y se aplica, con los matices propios del derecho del trabajo, a un contrato de trabajo de plazo fijo concluye que:

“...Condicionar la existencia y validez del fuero maternal al conocimiento que de este tenga el empleador, implica minimizar la institución de la protección a la maternidad, en base a principios propios del derecho civil, en el sentido de otorgarle mayor fuerza a la autonomía de la voluntad de las partes al razonar que ha sido la decisión de ellos los que fijaron un determinado plazo de duración del contrato, lo que tendría prioridad ante el embarazo, razonamiento que para esta Corte se contrapone a las normas constitucionales y laborales que ya se han expuesto..." (Considerando cuarto).

\section{ANÁLISIS DEL FALLO}

De la interpretación del tribunal se desprende una contradicción "aparente" entre normas legales, la que es constatada por la Corte de Apelaciones de Temuco y otra no evidenciada por el juzgador, cual dice relación con el conflicto entre institutos jurídicolaborales. En cuanto al primer conflicto normativo, la incoherencia es "aparente" pues, en el caso sub lite, se aplican debidamente las normas que regulan el fuero, y en particular el fuero maternal, aun cuando las reglas legales establezcan la existencia del contrato de plazo fijo. Como bien hemos señalado, la contradicción es "aparente" instituyen el fuero laboral y, en específico, las de protección a la maternidad, son aplicables a todos los trabajadores, indistintamente del tipo del contrato laboral de que se trate ${ }^{2}$, allegándose a una solución por aplicación de la norma laboral.

Ahora bien, la Corte soslayó una tensión más profunda y por ende más crítica: el conflicto entre dos instituciones del derecho del trabajo, a saber, el contrato de plazo fijo frente a las normas que regulan el fuero laboral ${ }^{3}$. En efecto, tal como lo señala la doctrina "el factor temporal incide en la tipología del contrato de trabajo, de tal manera que la distinta configuración temporal de la relación laboral da lugar a la construcción de distintos tipos contractuales" ${ }^{3}$. De ahí que, en los ordenamientos jurídicos laborales se consagren contratos temporales, en atención, entre otros factores, a la flexibilidad y movilidad de la economía del trabajo.

\footnotetext{
${ }^{1}$ DuCCI Claro, Interpretación juridica, tercera edición, Editorial Jurídica de Chile, Santiago, 2006, pp. 57-58.

2 Humeres Noguer, Héctor, Derecho del trabajo y de la Seguridad Social, Editorial Jurídica de Chile, Santiago, 2006.

${ }^{3}$ Esto es propio, al decir de Rodrigo Valenzuela Cori, del "discurso técnico", donde lo que se busca es hacer hablar al objeto, en este caso, la ley aplicable al caso concreto. Mostrar las cosas "tal cual son", sin entrar a rememorar y menos polemizar, públicamente, sobre sus consecuencias. VALENZUELA CORI, Rodrigo, Retórica. Un ensayo sobre tres dimensiones de la argumentación, Editorial Jurídica de Chile, Santiago, 2009, pp. 65-72.

${ }^{4}$ Montoya Melgar, Alfredo, Derecho del Trabajo, Editorial Tecnos, Madrid, 2004, p. 331.
} 
Por otra parte, las normas que regulan el fuero laboral y en especial el maternal, tienen como propósito proteger a "los trabajadores más débiles en razón de su edad, de sus sexo o de ciertas circunstancias que disminuyen por razones orgánicas su capacidad de trabajo, como son la enfermedad o el estado de gravidez"5. Es decir, apuntan a una clara protección de los trabajadores, pero junto a ello, han de otorgar una estabilidad en el empleo al extender, en algunos casos, sus efectos a una eventual desvinculación del trabajador $^{6}$.

En consecuencia, una aplicación de las reglas que regulan estos institutos jurídicos torna patente una contradicción, en la medida en que el primero -contrato a plazo fijopersigue una marcada transitoriedad del empleo, mientras que la segunda -fuero maternal-pretende precaver una eventual desvinculación del trabajador, entre otras situaciones, dado su especial estado o circunstancia por el cual atraviesa, consagrando de esta forma la estabilidad del empleo. Producto de esta tensión, se constata que los tribunales arriban a soluciones disímiles, no solo por la deficiente redacción de las disposiciones en juego, sino porque algunas sentencias han desconocido la aplicación del fuero maternal en los contratos de plazo $\mathrm{fijo}^{7}$, mientras que otras, como la sentencia analizada, aplica las normas del fuero maternal a la luz de una interpretación basada en los derechos fundamentales.

En segundo término, es necesario centrar nuestra atención en la argumentación presentada por el tribunal, relacionada con los derechos fundamentales, tanto en su aplicación como en el efecto de dichos derechos en las relaciones laborales. Para acometer dicha tarea es necesario presentar lo que la doctrina constitucional ha denominado eficacia hacia los terceros de los derechos fundamentales o Drittwirkung der Grundrechte, cuyo desarrollo conceptual ha sido fecundo tanto en Alemania como en España ${ }^{8}$. En síntesis, dicha doctrina señala que la aplicación de los derechos fundamentales no solo se reduciría a las relaciones entre el poder público y el individuo, sino que también sus efectos irradiarían a las relaciones entre particulares ${ }^{9}$. Esta idea gira en torno a dos versiones. La primera, denominada eficacia directa o inmediata, consiste en que los derechos fundamentales rigen automáticamente las relaciones entre particulares, siendo oponibles entre las partes, sin necesidad de mediación legal ni judicial que desarrollen dichos derechos. La segunda,

\footnotetext{
${ }^{5}$ HUMERES, op. cit., p. 254.

${ }^{6}$ Prueba de ello, es que la norma que regula el término del contrato respecto de los trabajadores sujetos a fuero laboral -art. 174- se encuentra en el Título V, denominado "De la terminación del contrato de trabajo y estabilidad en el empleo".

${ }^{7} \mathrm{La}$ Corte Suprema sentenció que "si el contrato de trabajo de la demandante era a plazo fijo, habiendo sido despedida a su vencimiento, se ajusta a derecho la sentencia que rechaza la demanda de nulidad de despido por fuero maternal. Se debe tener presente que la demandante no acreditó haber comunicado, ni informado al empleador durante la relación laboral y antes de su conclusión del trabajo del embarazo que tenía. El fuero maternal no puede invocarse para pretender prolongar indebidamente una relación laboral de muy corto plazo ya terminada, sobre todo que, en este caso, el embarazo fue dado a conocer una vez expirado el contrato, invocándose ese estado para pretender revivirlo". En: Corte Suprema, Rol No 1.013-2003, 23 de junio de 2003.

${ }^{8}$ STARCK, Christian, "Derechos fundamentales y derecho privado", en: Revista Española de Derecho Constitucional, año 22, No 66, septiembre-diciembre, 2002. Salmona MAUREIRA, Francisco José, Decisión judicial y colisión de derechos fundamentales, Cuadernos del Tribunal Constitucional, número 40, Santiago, 2008, passim.

9 AlEXY, Robert, Teoria de los derechos fundamentales, Madrid, 2007, pp. 464-468. En nuestro país, la idea fue inicialmente recogida por PENAA, Carlos, Sistema Jurídico y Derechos Humanos, Universidad Diego Portales, Santiago, 1996. Asimismo, JANA LINETZKY, Andrés, "La eficacia horizontal de los derechos fundamentales", en: SELA 2001, Seminario en Latinoamérica de Teoria Constitucional y Política, Editores del Puerto, Buenos Aires, 2003.
} 
llamada eficacia indirecta o mediata, los derechos fundamentales obligan única y exclusivamente a los poderes públicos, los que deben, en el ejercicio de sus funciones, aplicarlos a las relaciones entre particulares, mediatizados en leyes que los desarrollen o interpretaciones judiciales que los invoquen ${ }^{10}$.

En nuestro medio, dicha distinción se difumina dada la positivización constitucional de la eficacia directa de los derechos fundamentales ante los poderes públicos y entre los particulares, como bien lo señala el artículo $6^{\circ}$ de la Constitución ${ }^{11}$, al modo de las constituciones española y portuguesa ${ }^{12}$.

En el caso en cuestión, es posible advertir que el tribunal realizó una aplicación indirecta de los derechos fundamentales en juego, la que pudo haber hecho de manera inmediata, como se desprende de la Carta Fundamental. En este sentido y en consideración a la tensión de las normas laborales ya referidas, la argumentación desarrollada por la Corte, conforme con la Constitución, pese a ser un medio más para solucionar la controversia, no altera en lo absoluto la paradójica solución a la cual se arriba, la cual se obtiene, de igual forma, al aplicar los principios propios de la legislación laboral -principio protector- y las normas que emanan del Código del Trabajo y que, de forma implícita, refuerzan los derechos fundamentales señalados por la propia Corte. Sin duda, el tribunal recurre a los derechos fundamentales del trabajador - "los pesos pasados del sistema de derechos"como una forma de contrarrestar el impacto que provocaría una aplicación a secas del principio de la autonomía de la voluntad en materia laboral, así como para evitar un pronunciamiento sobre la tensión entre la institución del fuero maternal y el contrato de plazo fijo ${ }^{13}$.

\section{CONCLUSIÓN}

A partir de lo expuesto, es posible deducir la urgente necesidad de reformar y dar coherencia sistemática a nuestro actual Código del Trabajo, ya que debido a patentes

\footnotetext{
10 Ugarte Cataldo, José Luis, "La tutela de los derechos fundamentales y el derecho del trabajo: de erizo a zorro", en: Revista de Derecho, vol. XX No 2, Valdivia, diciembre 2007, pp. 56-57.

11 MARTf́nez EsTAY, José Ignacio, "Los particulares como sujetos pasivos de los derechos fundamentales: la doctrina del efecto horizontal de los derechos", en: Revista Chilena de Derecho, Universidad Católica, número especial, 1998.

12 En el caso de la normativa laboral, Ugarte Cataldo le reconoce tal eficacia inmediata pues señala: "En Chile, la eficacia horizontal ha sido plenamente acogida y en su versión más radical: la eficacia directa o inmediata. Reconocida, en efecto, bajo la denominación local de 'principio de vinculación directa de la Constitución', según los claros términos de su artículo $6^{\circ}$, inciso segundo, que señala que "los preceptos de esta Constitución obligan tanto a los titulares o integrantes de dichos órganos como a toda persona, institución o grupo". En: UGarTe CaTaldo, José Luis, op. cit. Por otra parte, constitucionalistas como Martínez Estay, aducen una aplicación de la eficacia horizontal pero con "sentido común", es decir, reconociendo la diferencia tanto en estructura, como en su lógica interna, de los derechos fundamentales como otros subsistemas jurídicos, sin reconocer una aplicación inmediata. A dicha conclusión arriban, por otra parte, iusprivatistas, como Pizarro Wilson y Jana Linetzky. Así, en palabras de Pizarro, al referirse al problema a resolver: "Intento concentrarme en la relación entre derecho constitucional y derecho privado, cuya interrelación, según se advierte en la literatura existente sobre el tema y en las decisiones de los tribunales, parece ser el punto de inflexión en el debate. A partir de ella defenderé la aplicación horizontal indirecta -en lugar de la directa- de los derechos fundamentales a las relaciones entre particulares", en: PIZARRO WILSON, Carlos, "Los derechos fundamentales y los contratos. Una mirada a la Drittwirkung”, en: Gaceta juridica, 221, 1998. JANA LiNETZKY, Andrés, op. cit.

13 VAlenzUela CORI, Rodrigo, op. cit.
} 
Roberto Cerón Reyes y Felipe Peroti Díaz / Una decisión controvertida: contrato de plazo fijo y fuero maternal

errores (u omisiones) de técnica legislativa y su constante adaptación a la realidad, provocan un sinnúmero de equívocos que impiden alcanzar la seguridad jurídica, imprescindible en cualquier estado de derecho. Esto tiene una explicación. El derecho del trabajo moderno, entendido como el ordenamiento que emergió frente a la necesidad de paliar la cruda realidad social imperante, a causa del primer capitalismo de corte industrial, entre otras variables, y que intenta regular la relación jurídica laboral entre empleados y empleadores, se ha caracterizado, constantemente, por actuar ante los hechos consumados, ya sea para crear, modificar o revocar una norma jurídica. Tantea, tal como lo hacen los maestros de cocina con sus ingredientes, ante la realidad ambigua, cambiante y escurridiza, dando con la solución circunstancial más adecuada. De ahí la indiscutible historicidad de esta rama del derecho ${ }^{14} \mathrm{y}$, como hemos constatado, sus variopintas instituciones.

Lo anterior, quizá permita entender la precaria -nula- regulación de los contratos de plazo fijo, los cuales sin un estatuto y reglas uniformes generan disonancias normativas como la analizada, obligando a los tribunales a recurrir, como es el caso, innecesariamente al sistema de derechos fundamentales o a un uso indiscriminado de los principios laborales, en ausencia de normas jurídico laborales claras ${ }^{15}$. Abogamos, tal cual acaece en otras situaciones $^{16}$, por una regulación especial del fuero en materia de contratos de plazo fijo.

\footnotetext{
${ }^{14}$ Decimos moderno, para hacer referencia a la regulación sistemática que comienza a emerger a mediados del siglo XIX en la vieja Europa capitalista y que, en Hispanoamérica, emerge a fines del mismo siglo. De este modo, no desconocemos la frondosa legislación indiana reguladora del trabajo indígena. BRAVO LIRA, Bernardino, Derecho común y derecho propio en el nuevo mundo, Editorial Jurídica de Chile, Santiago, 1989, y JARA, Álvaro, Trabajo y salario indigena siglo XVI, Editorial Universitaria, Santiago, 1987.

15 Decimos uso indiscriminado del principio protector, puesto que ha sido ampliamente utilizado por la jurisprudencia judicial para balancear situaciones, al menos, discutibles. Para un análisis del principio véase CERÓN REYES, Roberto, "Algunas reflexiones en torno al principio protector en el Derecho del Trabajo y su recepción en la jurisprudencia judicial", en: Actualidad Laboral, Facultad de Derecho, Universidad de Chile, diciembre 2008. Para los principios del derecho del trabajo es clásico Plá Rodrf́gueZ, Américo, Los principios del Derecho del Trabajo, tercera edición, Editorial Depalma, Buenos Aires, 1998.

${ }^{16} \mathrm{Tal}$ es el caso de la norma del artículo 183-AE, inciso primero, del Código del Trabajo, a propósito del fuero maternal de los trabajadores contratados por empresas de servicios transitorios -E.S.T.- y que han sido puestos en una empresa usuaria. En efecto, indica que "Las trabajadoras contratadas bajo el régimen contemplado en este Párrafo, gozarán del fuero maternal señalado en el inciso primero del artículo 201, cesando este de pleno derecho al término de los servicios en la usuaria". LIZAMA y UGARTE, si bien no justifican el espíritu de la norma, señalan que el sentido de este artículo es la transitoriedad del trabajador en la empresa usuaria. A nuestro juicio, algo similar ocurre con una gran cantidad de contratos a plazo fijo, lo que puede servir, con algunas precisiones y protecciones, como modelo. LIZAMA PORTAL, Luis y UGARTE CATALDO, José Luis, Subcontratación y suministro de trabajadores, Editorial LexisNexis, Santiago, 2007, p. 104.
} 